\title{
On Parameter Estimation Using Nonparametric Noise Models
}

\author{
Kaushik Mahata, Member, IEEE, Rik Pintelon, Fellow, IEEE, and Johan Schoukens, Fellow, IEEE
}

\begin{abstract}
Fitting multidimensional parametric models in frequency domain using nonparametric noise models is considered in this paper. A nonparametric estimate of the noise statistics is obtained from a finite number of independent data sets. The estimated noise model is then substituted for the the true noise covariance matrix in the maximum likelihood loss function to obtain suboptimal parameter estimates. The goal here is to present an analysis of the resulting estimates. Sufficient conditions for consistency are derived, and an asymptotic accuracy analysis is carried out. The first- and second-order statistics of the cost function at the global minimum point are also explored, which can be used for model validation. The analytical findings are validated using numerical simulation results.
\end{abstract}

Index Terms-Consistency, frequency domain, nonparametric noise models, multivariable models, multiple-input-multiple-output (MIMO) systems, statistical analysis, system identification.

\section{INTRODUCTION}

$\mathbf{C}$ ONSIDER the vector valued frequency domain data $\left\{\mathbf{y}\left[\omega_{\mathrm{k}}\right]\right\}_{\mathrm{k}=0}^{N-1}$, at $N$ discrete frequencies

$$
\omega_{\mathrm{k}}=\frac{2 \pi}{N} \quad \mathrm{k}, \mathrm{k}=0, \ldots, N-1 .
$$

Here the dimension of $\mathbf{y}\left[\omega_{\mathbf{k}}\right]$ is $p$ for all $\mathbf{k}$. It is further assumed that the data $\left\{\mathbf{y}\left[\omega_{\mathbf{k}}\right]\right\}_{\mathbf{k}=0}^{N-1}$ depend on an $n \times 1$ unknown vector valued parameter $\boldsymbol{\theta}$ through the relation

$$
\mathbf{y}\left[\omega_{\mathrm{k}}\right]=\mathbf{f}_{\mathrm{k}}(\boldsymbol{\theta}) .
$$

The functional forms of the relations $\left\{\mathbf{f}_{\mathbf{k}}(\boldsymbol{\theta})\right\}_{\mathrm{k}=0}^{N-1}$ are known. In this paper we consider vector valued functions $\left\{\mathbf{f}_{\mathrm{k}}(\boldsymbol{\theta})\right\}_{\mathrm{k}-0}^{N-1}$ with fairly general structure. Later, we will impose certain mild restrictions on the properties of $\left\{\mathbf{f}_{\mathrm{k}}(\boldsymbol{\theta})\right\}_{\mathrm{k}-0}^{N-1}$, see Assumptions 2,4 , and 5 . The central problem addressed in this paper is to estimate $\boldsymbol{\theta}$ from the noise corrupted observations of $\left\{\mathbf{y}\left[\omega_{\mathrm{k}}\right]\right\}_{\mathrm{k}=0}^{N-1}$.

Manuscript received February 26, 2004; revised January 29, 2005. Recommended by Associate Editor E. Bai. This work was supported by the Swedish Research Council; the Fund for Scientific Research (FWO-Vlaanderen), the Flemish Government (GOA-Ilinois) and the Belgian Program on Interuniversity Poles of Attraction initiated by the Belgian State, Prime Ministers Office, Science Policy programming (IUAP V/22).

K. Mahata is with the Centre for Complex Dynamic Systems and Control, University of Newcastle, Callaghan NSW-2308, Australia. (e-mail: Kaushik. Mahata@newcastle.edu.au).

R. Pintelon and J. Schoukens are with the Department ELEC, Vrije Universiteit Brussel, B-1050 Brussels, Belgium. (e-mail: Rik.Pintelon@vub.ac.be; Johan.Schoukens@vub.ac.be).

Digital Object Identifier 10.1109/TAC.2006.882936
We will assume $\boldsymbol{\theta}$ to be a complex valued parameter vector throughout this paper. Fitting parametric models in frequency domain is a subject of fundamental interest in many disciplines in science, see for instance [1]-[4]. Development of accurate measurement techniques in frequency domain in the past two decades [5], [6] has widened the scope of frequency domain model fitting. Several algorithms have been developed, see for example [7]-[11], which are being employed in various practical applications.

Given the frequency domain measurements the best (in terms of the statistical properties of the estimators) way to estimate the parameters is to employ the maximum likelihood estimator. Assume that for each $\mathrm{k}$, we observe noise corrupted measurement $\mathbf{z}\left[\omega_{\mathbf{k}}\right]$ of $\mathbf{y}\left[\omega_{k}\right]$ which is given by

$$
\mathbf{z}\left[\omega_{\mathbf{k}}\right]=\mathbf{y}\left[\omega_{\mathbf{k}}\right]+\tilde{\mathbf{y}}\left[\omega_{\mathbf{k}}\right] .
$$

where $\tilde{\mathbf{y}}\left[\omega_{\mathbf{k}}\right]$ is the measurement noise at frequency $\omega_{\mathbf{k}}$. Then, the maximum likelihood estimate of $\boldsymbol{\theta}$ is given by

$$
\begin{aligned}
\hat{\boldsymbol{\theta}}_{M L} & =\arg \min _{\mathbf{x}}\left[\min _{\boldsymbol{\beta}} \frac{1}{N} \sum_{\mathbf{k}=0}^{N-1} \hbar_{\mathbf{k}}(\mathbf{x})\right] \\
\hbar_{\mathbf{k}}(\mathbf{x}) & =\left\{\mathbf{z}\left[\omega_{\mathbf{k}}\right]-\mathbf{f}_{\mathbf{k}}(\mathbf{x})\right\}^{*} \mathbf{\Sigma}_{\mathbf{k}}^{-1}(\boldsymbol{\beta})\left\{\mathbf{z}\left[\omega_{\mathbf{k}}\right]-\mathbf{f}_{\mathbf{k}}(\mathbf{x})\right\}
\end{aligned}
$$

where $\mathrm{x}$ is the dummy variable of optimization, and $\boldsymbol{\Sigma}_{\mathrm{k}}(\boldsymbol{\beta})$ is the covariance matrix of $\tilde{\mathbf{y}}\left[\omega_{k}\right]$ parameterized by the vector valued parameter $\boldsymbol{\beta}$. In general, in a practical application the noise covariance matrix is unknown. ${ }^{1}$ In such cases, it is a common practice in system identification and signal processing literature to use such parameterizations ${ }^{2}$ of the noise covariance matrices [12], [13]. Note that the size of the parameter vector $\beta$ required for a multidimensional model can be significantly large. As a result, the dimension of the search space in the optimization problem (4) grows rapidly with increase in the dimension. Secondly, it might not be reasonable to use such parameterization. Therefore, it is often preferred to use a nonparametric noise model [14]-[16]. In this case we estimate the second order noise statistics using a finite number of observations at every frequency, and use the nonparametric estimate of the noise covariance matrix instead of the parameterized noise

${ }^{1}$ If the noise covariance matrix is known to the user, we need not minimize the loss function in (4) with respect to $\boldsymbol{\beta}$.

${ }^{2}$ Since the additive noise is generally independent of the data, it is reasonable to use independent parameters for the transfer function model and the noise model. Therefore, we optimize over two different mutually independent parameters $\mathbf{x}$ and $\boldsymbol{\beta}$ in (4). 
covariance matrix $\boldsymbol{\Sigma}_{\mathbf{k}}(\boldsymbol{\beta})$. The key here is to use a periodic input excitation, commonly known as a multisine excitation, with a known period. However, the measured data is not exactly periodic. In each period, the deterministic part $\mathbf{y}\left[\omega_{k}\right]$ of the measurement remains the same, but the measurement noise $\tilde{\mathbf{y}}\left[\omega_{\mathbf{k}}\right]$ varies. Therefore, measurements at each frequency at different periods constitute an independent and identically distributed sequence of random variables. ${ }^{3}$ From $M$ periods of the excitation, we can now extract the second order statistics of the measurement noise. Now the estimated covariance matrix $\hat{\boldsymbol{\Sigma}}_{\mathbf{k}}$ can be used instead of $\boldsymbol{\Sigma}_{\mathbf{k}}(\boldsymbol{\beta})$ in the loss function (4), and the resulting optimization problem is simplified.

Intuitively, one would expect for large $M$ (i.e., for large number of periods) the estimate using the nonparametric noise model will perform as good as the asymptotically efficient estimator. But from a practical point of view, one would like to use a low value of $M$ and still get a good estimate. Therefore, it is natural to ask how much do we loose in accuracy by using a nonparametric noise model, i.e., what is the relative efficiency of the suboptimal estimator obtained using the nonparametric noise model. It is also important to know the minimum value of $M$ for which we get a consistent estimator as $N \rightarrow \infty$. These are the main questions to be addressed in this paper. We will derive the conditions for consistency and explicit expressions for the asymptotic $(N \rightarrow \infty)$ covariance matrix of the parameter estimates for any finite $M$. We shall also explore the properties of the loss function at its global minimum, which helps the user to decide whether the model (2) is correct or not. A similar discussion regarding identification of single-input-single-output (SISO) dynamic errors-in-variables models was presented in [14]. The results presented in this paper can be seen as multi-dimensional generalizations of the results presented in [14]. We will show that all results given in [14] can be derived as a special case of the results in this paper by setting the dimension $p$ of $\mathbf{y}\left[\omega_{\mathbf{k}}\right]$ to unity.

This paper is organized follows. In the next section we present a mathematical description of the estimator considered in this paper. In Section III we derive the conditions for consistency of the parameter estimates, followed by the an accuracy analysis. We also derive the statistical properties of the loss function at the global minimum. In Section IV, we shall present a few simulation results, which will be used to validate the analytical results followed by the conclusions in Section V.

\section{ESTIMATION USING NONPARAMETRIC NOISE MODEL}

Assume that we observe $M$ independent data sets corresponding to $M$ periods of the multisine excitation signal, where in the ith data set the observation $\mathbf{y}_{\mathbf{i}}\left[\omega_{\mathbf{k}}\right]$ at frequency $\omega_{\mathrm{k}}$ is given by

$$
\mathbf{y}_{\mathrm{i}}\left[\omega_{\mathrm{k}}\right]=\mathbf{y}\left[\omega_{\mathrm{k}}\right]+\tilde{\mathbf{y}}_{\mathrm{i}}\left[\omega_{\mathrm{k}}\right] \text {. }
$$

\footnotetext{
${ }^{3}$ The reader should be aware that this is an approximation since in case of coloured noise the noise on the consecutive periods is correlated. The correlation is small if the correlation length is small compared to the period of the excitation. For this reason the number of periods are kept constant when the measurement time is increased. As a result, the number of samples in each period is increased which in turn increases the resolution in frequency domain. Thus, the number of frequency points in the frequency band of interest is increased.
}

Therefore, for each discrete frequency $\omega_{\mathrm{k}}$ we get $M$ independent noise corrupted measurements of $\mathbf{y}\left[\omega_{k}\right]$. We emphasize here that the frequency domain measurements mentioned previously are not the frequency response measurements of the observed time series, but they are actual measurements of the Fourier coefficients. Such measurements can be done using a periodic multisine excitation and discrete Fourier transform (DFT) techniques [5], [6]. During such measurements one must take special precautions to avoid transient effects and measurements can start only after the transients become negligible in magnitude. The spectral leakage phenomenon is avoided by measuring an integer number of samples in each period, while the aliasing problem can be handled using a suitable antialiasing filter. Here, we avoid the details and refer the interested readers to [5] and [6]. We have the following assumption on the measurement noise.

Assumption 1: The frequency domain noise sequence is Gaussian distributed and has zero mean, i.e.,

$$
\mathbf{E} \tilde{\mathbf{y}}_{\mathbf{i}}\left[\omega_{\mathbf{k}}\right]=0_{p \times 1}
$$

where $\mathbf{E}(\cdot)$ denotes the expectation operator. It is further assumed for all $i$ that $\tilde{\mathbf{y}}_{\mathbf{i}}\left[\omega_{k}\right]$ is independent of $\tilde{\mathbf{y}}_{\mathbf{i}}\left[\omega_{\mathrm{j}}\right]$ if $\mathbf{k} \neq \mathbf{j}$, and

$$
\left.\begin{array}{r}
\mathbf{E} \tilde{\mathbf{y}}_{\mathbf{j}}\left[\omega_{\mathbf{k}}\right] \tilde{\mathbf{y}}_{\mathbf{i}}^{*}\left[\omega_{\mathbf{k}}\right]=\boldsymbol{\Sigma}_{\mathbf{k}} \\
\mathbf{E} \tilde{\mathbf{y}}_{\mathbf{i}}\left[\omega_{\mathbf{k}}\right] \tilde{\mathbf{y}}_{\mathbf{i}}^{\top}\left[\omega_{\mathbf{k}}\right]=0_{p \times p}
\end{array}\right\} \mathbf{k}=0, \ldots, N-1 .
$$

Therefore, $\tilde{\mathbf{y}}_{\mathrm{i}}\left[\omega_{\mathrm{j}}\right]$ circular.

Assumption 1 is common and justifiable in most practical circumstances. If $N \rightarrow \infty$, we can prove the validity of Assumption 1 after a DFT. For practical data records this is a very good approximation. We omit the details here and refer the interested reader to [6], [17]. However, we point out that Assumption 1 is equivalent of having the underlying sampled noise sequence in time domain 4 to be zero mean with a spectral density $\boldsymbol{\Sigma}_{\mathrm{k}}$ at frequency $\omega_{\mathbf{k}}$.

The main idea here is to estimate the nonparametric noise model from the $M$ independent data sets. This means that we estimate $\left\{\boldsymbol{\Sigma}_{\mathbf{k}}\right\}_{\mathrm{k}=0}^{N-1}$ and substitute for $\left\{\boldsymbol{\Sigma}_{\mathbf{k}}(\boldsymbol{\beta})\right\}_{\mathbf{k}=0}^{N-1}$ in the likelihood cost function (4). At the same time we can also replace the measurement $\mathbf{z}\left[\omega_{\mathrm{k}}\right]$ at frequency $\omega_{k}$ by the sample mean from $M$ independent data sets. To that aim, let us define

$$
\begin{aligned}
& \overline{\mathbf{y}}_{\mid}=\frac{1}{M} \sum_{\mathbf{i}=1}^{M} \mathbf{y}_{\mathbf{i}}\left[\omega_{\mid}\right] \\
& \hat{\mathbf{\Sigma}}_{\mathbf{l}}=\frac{1}{M-1} \sum_{\mathbf{i}=1}^{M}\left\{\mathbf{y}_{\mathbf{i}}\left[\omega_{\mathbf{l}}\right]-\overline{\mathbf{y}}_{\mathbf{l}}\right\}\left\{\mathbf{y}_{\mathbf{i}}\left[\omega_{\mathbf{l}}\right]-\overline{\mathbf{y}}_{\mid}\right\}^{*} .
\end{aligned}
$$

Now in order to estimate $\boldsymbol{\theta}$, we can minimize the loss function

$$
\hat{\ell}_{N}(\mathbf{x})=\frac{1}{N} \sum_{\mathrm{l}=0}^{N-1} \lambda_{\mathrm{l}}(\mathbf{x})
$$

${ }^{4}$ Note that it is not needed to specify the distribution of the corresponding time domain noise. The frequency domain noise can be shown to be circular complex normally distributed under very mild assumptions [6], [17]. 
with respect to $\mathbf{x}$, where

$$
\begin{aligned}
\lambda_{\mathbf{I}}(\mathbf{x}) & =\left\{\overline{\mathbf{y}}_{\mid}-\mathbf{f}_{\mathbf{I}}(\mathbf{x})\right\}^{*} \hat{\boldsymbol{\Sigma}}_{\mid}^{-1}\left\{\overline{\mathbf{y}}_{\mid}-\mathbf{f}_{\mathbf{I}}(\mathbf{x})\right\} \\
& =\operatorname{Tr}\left[\hat{\boldsymbol{\Sigma}}_{\mid}^{-1}\left\{\overline{\mathbf{y}}_{\mid}-\mathbf{f}_{\mathbf{I}}(\mathbf{x})\right\}\left\{\overline{\mathbf{y}}_{\mid}-\mathbf{f}_{\mathbf{I}}(\mathbf{x})\right\}^{*}\right]
\end{aligned}
$$

Thus, we obtain an alternative estimate of $\boldsymbol{\theta}$ as

$$
\hat{\boldsymbol{\theta}}_{N}=\arg \min _{\mathbf{x}} \hat{\ell}_{N}(\mathbf{x})
$$

The approach in (12) is suboptimal. However, it gives several advantages listed below.

1) Using periodic excitation signals (or independent repeated experiments) and the pre-processing steps (8) and (9) we get the noise (co-)variances straightaway, which is then used as weighting in the parametric plant (or signal) modeling step. Therefore, there is no need of parametric modeling of the noise process and associated model selection.

2) The most popular way of parameterizing the noise model is to assume that the spectrum of the noise process is rational, see for example [12], [13]. For multivariable noise processes the number of parameters needed for such parameterization can be large, which causes a significant increase in computational demand in the numerical optimization step. Using a nonparametric noise model in (8)-(9), on the other hand, reduces the dimension of the search space. Therefore, the complexity of the numerical optimization step is reduced significantly. Second, the nonparametric noise model is more general, i.e., we need not impose any constraint on the noise spectral density.

3) As we shall show in Section III-D, the nonparametric noise model also allows to interpret the cost function in an absolute way, and hence to detect model errors which are hidden in the classical prediction error approach, e.g., nonlinear distortions.

4) Besides the frequency response function, one also has its uncertainty. This provide a way of easy model validation.

Intuitively, we must impose certain constraints on the number of data sets $M$ in order to guarantee the consistency of the parameter estimates for large $N$. It is also interesting to compare the asymptotic covariance matrix of $\hat{\boldsymbol{\theta}}_{N}$ to the Cramér-Rao bound. Therefore, in the rest of this paper we address, the following issues.

1) What is the minimum value of $M$ required to obtain any meaningful estimate of $\boldsymbol{\theta}$ ? An answer to this question provides user with an important guideline.

2) How much do we loose in the accuracy by using the suboptimal estimate in (12)? An answer to this question helps us to judge the quality of the estimates.

3) What are the statistics of the minimum value of the cost function in (12) if there is no model error? An answer to this question help us to detect the presence of any model error by just looking at the minimum value of the cost function in (12).

\section{ANALYSIS}

\section{A. Few Statistical Results}

In order to proceed further, we need a few preliminary results regarding the distribution of the sample means $\left\{\overline{\mathbf{y}}_{\mathbf{i}}\right\}_{\mathbf{i}=0}^{N-1}$ and sample covariance matrices $\left\{\hat{\boldsymbol{\Sigma}}_{\mathbf{i}}\right\}_{\mathbf{i}=0}^{N-1}$. By Assumption 1, $\tilde{\mathbf{y}}_{\mathbf{i}}$ is complex Gaussian with a covariance matrix $\boldsymbol{\Sigma}_{\mathbf{j}}$. The following proposition is a consequence of that.

Proposition 1: The sample mean

$$
\overline{\mathbf{y}}_{\mathbf{i}} \sim \mathcal{C N}\left\{\mathbf{y}_{\mathbf{i}}, \frac{1}{M} \boldsymbol{\Sigma}_{\mathbf{i}}\right\}
$$

where $\mathcal{C N}\left\{\mathbf{y}_{\mathbf{i}},(1 / M) \boldsymbol{\Sigma}_{\mathrm{i}}\right\}$ denotes the complex Gaussian distribution with mean $\mathbf{y}_{\mathbf{j}}$ and covariance matrix $(1 / M) \boldsymbol{\Sigma}_{\mathbf{j}}$. The estimated covariance matrix

$$
\hat{\boldsymbol{\Sigma}}_{\mathrm{i}} \sim \mathcal{C} \mathcal{W}_{p}\left\{M-1, \frac{1}{M-1} \boldsymbol{\Sigma}_{\mathrm{i}}\right\}
$$

where $\mathcal{C W}_{p}(K, \mathbf{S})$ denotes the complex Wishart distribution of dimension $p$, degree of freedom $K$ and associated $p \times p$ covariance matrix $\mathbf{S}$. Furthermore, $\overline{\mathbf{y}}_{\mathrm{i}}$ and $\hat{\boldsymbol{\Sigma}}_{\mathrm{i}}$ are statistically independent of each other.

Proof: It is straightforward to derive (13) from Assumption 1. The complex Wishart distribution was defined in [18], where (14) was also established. Mutual independence of the sample mean $\overline{\mathbf{y}}_{\mathbf{j}}$ and the sample covariance matrix $\hat{\boldsymbol{\Sigma}}_{\mathbf{j}}$ is also well known, see [19] for example.

The results in Proposition 1 will be used extensively in the statistical analysis presented in the rest of this paper. As a direct consequence of (14), we have $\hat{\Sigma}_{\mathrm{i}}^{-1}$ to be complex inverse Wishart distributed [20] for all $\mathrm{i}$ such that $0 \leq \mathrm{i} \leq N-1$. In the following proposition, we summarize a few moment results regarding complex inverse Wishart distribution.

Proposition 2: Let the $p \times p$ Hermitian matrix $\hat{\mathbf{S}}$ be complex Wishart distributed with $k$ degrees of freedom

$$
\hat{\mathbf{S}} \sim \mathcal{C W}_{p}(k, \mathbf{S})
$$

and, hence, the matrix $\hat{\mathbf{S}}^{-1}$ is complex inverse Wishart distributed. Then the expected value $\mathbf{E} \hat{\mathbf{S}}^{-1}$ exists if $k-p>0$ and is given by

$$
\mathbf{E} \hat{\mathbf{S}}^{-1}=\frac{1}{k-p} \mathbf{S}^{-1}, k>p .
$$

Assume further that the matrices $\mathbf{S}$ and $\hat{\mathbf{S}}^{-1}$ are given elementwise as

$$
\mathbf{S}^{-1}=\left(s_{\mathrm{ij}}\right), \hat{\mathbf{S}}^{-1}=\left(\hat{s}_{\mathrm{ij}}\right)
$$


then the second-order moment $\mathbf{E}\left\{\hat{s}_{\mathrm{ij}} \hat{s}_{\mathrm{kl}}\right\}$ exists if $k-p-1>0$ and is given by

$$
\mathbf{E}\left\{\hat{s}_{\mathrm{ij}} \hat{s}_{\mathrm{kl}}\right\}=\frac{1}{(k-p)^{2}-1}\left\{s_{\mathrm{ij}} s_{\mathrm{kl}}+\frac{1}{k-p} s_{\mathrm{ik}} s_{\mathrm{lj}}\right\} \text {. }
$$

Proof: The proof of these results are given in [20]. See also [21] for a related discussion on the real inverse Wishart distribution.

If we have had scalar valued observations, i.e., if each of $\left\{\mathbf{y}_{\mathrm{i}}\left[\omega_{\mathrm{k}}\right]\right\}_{\mathrm{k}=1}^{M}$ is scalar valued, then the random variable $(M-$ 1) $\boldsymbol{\Sigma}^{-1} \hat{\boldsymbol{\Sigma}}$ would be chi-square distributed with $2 M-2$ degrees of freedom. From the property of the chi-square distribution, it can be shown using the moment generating function of the chi-square distribution [22] that

$$
\begin{aligned}
\mathbf{E} \hat{\boldsymbol{\Sigma}}^{-1} & =\frac{M-1}{M-2} \boldsymbol{\Sigma}^{-1} \\
\mathbf{E} \hat{\boldsymbol{\Sigma}}^{-2} & =\frac{(M-1)^{2}}{(M-2)(M-3)} \boldsymbol{\Sigma}^{-2} .
\end{aligned}
$$

These results are in prefect agreement with the results given in Proposition 2 using $p=1$. In fact it can be shown [18] that the Wishart distribution is the multivariable generalization of the chi-square distribution.

\section{B. Consistency}

In this section, we establish the conditions for the strong consistency of the parameter estimate $\hat{\boldsymbol{\theta}}_{N}$ in absence of any model error. We emphasize at this point that in the analysis to be presented here, we do not assume the model (2). Therefore, the following analysis is valid even in presence of any model error [i.e., when (2) does not hold]. We have the following proposition as a consequence of Proposition 1.

Proposition 3: Assume that the number of data sets $M>$ $p+1$. Let us define

$$
\ell_{N}(\mathbf{x}):=\mathbf{E} \hat{\ell}_{N}(\mathbf{x}), \mathbf{h}_{\mathbf{i}}(\mathbf{x}):=\mathbf{y}_{\mathbf{i}}-\mathbf{f}_{\mathbf{i}}(\mathbf{x}) .
$$

Then under Assumption $1, \ell_{N}(\mathbf{x})$ exists for all $\mathbf{x}$ and is given by

$$
\ell_{N}(\mathbf{x})=\frac{M-1}{K N} \sum_{\mathbf{i}=0}^{N-1}\left[\mathbf{h}_{\mathbf{i}}^{*}(\mathbf{x}) \boldsymbol{\Sigma}_{\mathbf{i}}^{-1} \mathbf{h}_{\mathbf{i}}(\mathbf{x})+\frac{p}{M}\right]
$$

where we have introduced

$$
K=M-p-1 .
$$

Proof: Let us introduce the noise contribution to the sample mean $\overline{\mathbf{y}}_{\boldsymbol{i}}$ as

$$
\check{\mathbf{y}}_{\mathbf{i}}:=\overline{\mathbf{y}}_{\mathbf{i}}-\mathbf{y}_{\mathbf{i}}, \mathrm{i}=0, \ldots, N-1 \text {. }
$$

Therefore from (13) it is straightforward that

$$
\check{\mathbf{y}}_{\mathbf{i}} \sim \mathcal{C N}\left\{0_{p \times 1}, \frac{1}{M} \Sigma_{\mathrm{i}}\right\}, \mathrm{i}=0, \ldots, N-1 .
$$

Now, from (20) and (23), we have

$$
\overline{\mathbf{y}}_{\mathrm{i}}-\mathbf{f}_{\mathbf{i}}(\mathbf{x})=\mathbf{h}_{\mathrm{i}}(\mathbf{x})+\check{\mathbf{y}}_{\mathrm{i}} \sim \mathcal{C N}\left\{\mathbf{h}_{\mathrm{i}}(\mathbf{x}), \frac{1}{M} \boldsymbol{\Sigma}_{\mathrm{i}}\right\} .
$$

Again from (16) in Proposition 2, we see that

$$
\mathbf{E} \hat{\Sigma}_{\mathbf{i}}^{-1}=\frac{M-1}{K} \boldsymbol{\Sigma}_{\mathbf{i}}^{-1}, K>0 .
$$

Since $\overline{\mathbf{y}}_{\mathbf{j}}$ and $\hat{\boldsymbol{\Sigma}}_{\mathbf{j}}$ are independent of each other, it follows that $\check{\mathbf{y}}_{\mathbf{j}}$ and $\hat{\Sigma}_{\mathbf{j}}$ are also independent of each other. Therefore, when we compute the expectation of any function of $\check{\mathbf{y}}_{\mathfrak{j}}$ and $\hat{\boldsymbol{\Sigma}}_{\mathfrak{j}}$, the expectation computations can be carried out independently with respect to each of them. Therefore, from (11), (24) and (26) we get for all $K>0$

$$
\begin{aligned}
\mathbf{E} \lambda_{\mathbf{i}}(\mathbf{x}) & =\frac{M-1}{K} \operatorname{Tr}\left[\boldsymbol{\Sigma}_{\mathbf{i}}^{-1} \mathbf{h}_{\mathbf{i}}(\mathbf{x}) \mathbf{h}_{\mathbf{i}}^{*}(\mathbf{x})+\frac{1}{M} \boldsymbol{\Sigma}_{\mathbf{i}}^{-1} \boldsymbol{\Sigma}_{\mathbf{i}}\right] \\
& =\frac{M-1}{K}\left[\mathbf{h}_{\mathbf{i}}^{*}(\mathbf{x}) \boldsymbol{\Sigma}_{\mathbf{i}}^{-1} \mathbf{h}_{\mathbf{i}}(\mathbf{x})+\frac{p}{M}\right] .
\end{aligned}
$$

Now, recall that

$$
\ell_{N}(\mathbf{x}):=\mathbf{E} \hat{\ell}_{N}(\mathbf{x})=\frac{1}{N} \sum_{\mathrm{k}=0}^{N-1} \mathbf{E} \lambda_{\mathbf{k}}(\mathbf{x}) .
$$

Thus, combining (27) and (28), the proposition follows.

At our next step, we establish strong uniform convergence of the loss function $\hat{\ell}_{N}(\mathbf{x})$ to its expected value. In order to proceed further, we need the following assumption.

Assumption 2: There exists a compact and dense set $\mathcal{S} \subset \mathbb{C}^{n}$ such that $\left|\mathbf{f}_{\mathbf{i}}(\mathbf{x})\right|$ is uniformly bounded for all $\mathbf{x} \in \mathcal{S}$ and all $\mathrm{i}$ such that $0 \leq \mathrm{i} \leq N-1$.

Proposition 4: Under Assumptions 1 and 2,

$$
\lim _{N \rightarrow \infty} \hat{\ell}_{N}(\mathbf{x})-\ell_{N}(\mathbf{x})=0
$$

uniformly with probability one for all $\mathrm{x} \in \mathcal{S}$ provided the number of data sets $M>p+2$.

Proof: Since the terms in the summation (10) are independent of each other, convergence with probability one in (29) is implied if the convergence is established in mean square sense [23], [24]. Toward that goal, it will be sufficient to show for every $k$ that the variance of $\lambda_{k}(\mathbf{x})$ is uniformly bounded for all $\mathbf{x} \in \mathcal{S}$. In that aim we first compute $\mathbf{E} \lambda_{\mathbf{k}}^{2}(\mathbf{x})$. First, we take the expectation with respect to $\check{\mathbf{y}}_{\mathrm{k}}$ and denote the associated operator by $\mathbf{E}_{\check{y}_{\mathbf{k}}}(\cdot)$. Since the third-order moments of a zero mean 
Gaussian random vector vanish, we have using (11) and (24) after a few steps of algebra

$$
\begin{aligned}
& \mathbf{E}_{\check{\mathbf{y}}_{\mathbf{k}}} \lambda_{\mathrm{k}}^{2}(\mathbf{x}) \\
& =\mathbf{E}_{\check{\mathbf{y}}_{\mathbf{k}}}\left[\left\{\mathbf{h}_{\mathbf{k}}^{*}(\mathbf{x}) \hat{\boldsymbol{\Sigma}}_{\mathbf{k}}^{-1} \mathbf{h}_{\mathbf{k}}(\mathbf{x})\right\}^{2}\right. \\
& +2 \mathbf{h}_{\mathrm{k}}^{*}(\mathbf{x}) \hat{\boldsymbol{\Sigma}}_{\mathrm{k}}^{-1} \check{\mathbf{y}}_{\mathrm{k}} \check{\mathbf{y}}_{\mathrm{k}}^{*} \hat{\boldsymbol{\Sigma}}_{\mathrm{k}}^{-1} \mathbf{h}_{\mathrm{k}}(\mathbf{x}) \\
& \left.+\left\{\check{\mathbf{y}}_{\mathbf{k}}^{*} \hat{\boldsymbol{\Sigma}}_{\mathbf{k}}^{-1} \check{\mathbf{y}}_{\mathbf{k}}\right\}^{2}+2 \mathbf{h}_{\mathbf{k}}^{*}(\mathbf{x}) \hat{\boldsymbol{\Sigma}}_{\mathbf{k}}^{-1} \mathbf{h}_{\mathbf{k}}(\mathbf{x}) \check{\mathbf{y}}_{\mathbf{k}}^{*} \hat{\boldsymbol{\Sigma}}_{\mathbf{k}}^{-1} \check{\mathbf{y}}_{\mathbf{k}}\right] \\
& =\left\{\mathbf{h}_{\mathbf{k}}^{*}(\mathbf{x}) \hat{\mathbf{\Sigma}}_{\mathrm{k}}^{-1} \mathbf{h}_{\mathbf{k}}(\mathbf{x})\right\}^{2} \\
& +\frac{1}{M^{2}}\left[\operatorname{Tr}\left\{\hat{\boldsymbol{\Sigma}}_{\mathrm{k}}^{-1} \boldsymbol{\Sigma}_{\mathbf{k}} \hat{\boldsymbol{\Sigma}}_{\mathrm{k}}^{-1} \boldsymbol{\Sigma}_{\mathrm{k}}\right\}+\operatorname{Tr}^{2}\left\{\hat{\boldsymbol{\Sigma}}_{\mathrm{k}}^{-1} \boldsymbol{\Sigma}_{\mathbf{k}}\right\}\right] \\
& +\frac{2}{M} \mathbf{h}_{\mathbf{k}}^{*}(\mathbf{x}) \hat{\boldsymbol{\Sigma}}_{\mathrm{k}}^{-1} \boldsymbol{\Sigma}_{\mathbf{k}} \hat{\boldsymbol{\Sigma}}_{\mathrm{k}}^{-1} \mathbf{h}_{\mathrm{k}}(\mathbf{x}) \\
& +\frac{2}{M} \mathbf{h}_{\mathbf{k}}^{*}(\mathbf{x}) \hat{\boldsymbol{\Sigma}}_{\mathbf{k}}^{-1} \mathbf{h}_{\mathrm{k}}(\mathbf{x}) \operatorname{Tr}\left\{\hat{\boldsymbol{\Sigma}}_{\mathbf{k}}^{-1} \boldsymbol{\Sigma}_{\mathbf{k}}\right\}
\end{aligned}
$$

where in the last equality we have used a well known formula [25] for computing the fourth order moment of a Gaussian random vector. Next, we take the expectation with respect to $\hat{\boldsymbol{\Sigma}}_{\mathbf{k}}$. From (26), Proposition 2 and the moment results in Appendix we get for all $K>1$,

$$
\begin{aligned}
\mathbf{E} \lambda_{\mathbf{k}}^{2}(\mathbf{x})= & \frac{(M-1)^{2}}{K(K-1)}\left[\frac{1}{M^{2}}\left(p^{2}+p\right)\right. \\
& +\frac{2}{M}(p+1) \mathbf{h}_{\mathbf{k}}^{*}(\mathbf{x}) \boldsymbol{\Sigma}_{\mathbf{k}}^{-1} \mathbf{h}_{\mathbf{k}}(\mathbf{x}) \\
& \left.+\left\{\mathbf{h}_{\mathbf{k}}^{*}(\mathbf{x}) \boldsymbol{\Sigma}_{\mathbf{k}}^{-1} \mathbf{h}_{\mathbf{k}}(\mathbf{x})\right\}^{2}\right] .
\end{aligned}
$$

Now, from Assumption 2, it is evident that $\mathbf{E} \lambda_{\mathbf{k}}^{2}(\mathbf{x})$ is uniformly bounded for all $\mathbf{x} \in \mathcal{S}$ as long as $K>1$. Since $\mathbf{E} \lambda_{\mathbf{k}}^{2}(\mathbf{x}) \geq$ $\operatorname{Var}\left\{\lambda_{\mathbf{k}}(\mathbf{x})\right\}$, the proposition follows.

Proposition 5: Let the sequence of functions $\left\{\mathcal{L}_{N}(\theta)\right\}$ be uniformly convergent (as $N \rightarrow \infty$ ) to a continuous function $\mathcal{L}_{\infty}(\theta)$ on a compact set $\Omega$, and let $\mathcal{L}_{\infty}(\theta)$ have a unique global minimum point at $\theta_{*}$. Let $\hat{\theta}_{N}$ be a global minimum point of $\mathcal{L}_{N}(\theta)$ in $\Omega$. Then $\hat{\theta}_{N}$ converges to $\theta_{*}$ as $N \rightarrow \infty$.

Proof: See [26] and [27].

Assumption 3: Let us define

$$
\boldsymbol{\theta}_{N *}:=\arg \min _{\mathbf{x} \in \mathcal{S}} \ell_{N}(\mathbf{x})
$$

which is the unique global minimum point of $\ell_{N}(\mathbf{x})$ in $\mathcal{S}$. Moreover, $\boldsymbol{\theta}_{N *}$ is an interior point of $\mathcal{S}$.

Proposition 5 is well known and is often used in signal processing and system identification literature in conjunction with an assumption like Assumption 3. Assumption 3 is an identifiability condition. Such an assumption is very common in system identification literature. We also emphasize that the global minimum point $\boldsymbol{\theta}_{N *}$ is $N$-dependent. This is because the model (2) may not be a correct one. In such a case, we have modeling errors. In presence of the model error, the global minimum point $\boldsymbol{\theta}_{N *}$ varies with $N$, and will not necessarily converge in general as $N \rightarrow \infty$. However, if the model (2) is correct then for all $N$ from (21) we can see that

$$
\ell_{N}(\mathbf{x}) \geq \frac{p(M-1)}{K M}=\ell_{N}(\boldsymbol{\theta})
$$

since $\Sigma_{\mathrm{j}}$ is nonnegative definite for all $\mathrm{i}$. Therefore, in the absence of any model error

$$
\arg \min _{\mathbf{x}} \ell_{N}(\mathbf{x})=\boldsymbol{\theta} \quad \forall N .
$$

We are now ready to state the main result of this section.

Proposition 6: Let the number of data sets $M>p+2$. Then under Assumptions 1-3

$$
\lim _{N \rightarrow \infty} \hat{\boldsymbol{\theta}}_{N}-\boldsymbol{\theta}_{N *}=\mathbf{0}_{n \times 1}
$$

with probability one.

Proof: The proposition follows using 5 Assumption 3, and by combining Propositions 4 and 5 .

In presence of model errors, therefore, it is not guaranteed to have a convergence of the parameter estimate $\hat{\boldsymbol{\theta}}_{N}$. However, if the model is correct then we can see from Proposition 6 that $\hat{\boldsymbol{\theta}}_{N} \rightarrow \boldsymbol{\theta}$ with probability one $N \rightarrow \infty$, provided $M>p+2$.

\section{Accuracy Analysis}

In this section, we explore the second order statistical properties of the estimates. Let us first introduce some further notations. Since the statistical properties of a complex valued quantity is usually expressed in terms of the joint statistical properties of its real and imaginary parts, it is convenient to use an associated real valued parameter vector. Let the true parameter vector $\boldsymbol{\theta}$ and the dummy parameter vector $\mathbf{x}$ be expressed in terms of their real and imaginary parts as

$$
\boldsymbol{\theta}=\boldsymbol{\theta}_{R}+\mathrm{i} \boldsymbol{\theta}_{I}, \quad \mathbf{x}=\mathbf{x}_{R}+\mathrm{i} \mathbf{x}_{I} .
$$

Following the usual convention, the joint statistical properties of the estimate $\hat{\boldsymbol{\theta}}_{N}$ will be expressed in terms of the statistical properties of the estimates of the real valued vectors

$$
\boldsymbol{\alpha}:=\left[\begin{array}{c}
\boldsymbol{\theta}_{R} \\
\boldsymbol{\theta}_{I}
\end{array}\right] \quad x:=\left[\begin{array}{c}
\mathbf{x}_{R} \\
\mathbf{x}_{I}
\end{array}\right] .
$$

Moreover, we will use $\hat{\boldsymbol{\alpha}}_{N}$ to denote the estimate of $\boldsymbol{\alpha}$ derived from $\hat{\boldsymbol{\theta}}_{N}$, etc. Note that, the mapping from the complex valued parameter vector $\mathbf{x}$ to the associated real valued parameter vector $\boldsymbol{x}$ is bijective: Any function of $\mathbf{x}$ can equivalently be expressed as functions of the associated real valued parameter vector $x$. With a slight misuse of notation, we maintain the same functional symbol to denote the equivalent function also. For example, we use $\ell_{N}(\boldsymbol{x})=\ell_{N}(\mathbf{x})$ and so on. Similarly the

${ }^{5}$ The proof is valid if some constraints are imposed on the way the frequencies are added in the experiment with the increase in $N$. One has to assume that the frequency density tends to some continuous distribution as $N \rightarrow \infty$. See [6] Assumption 7.14 on page 194 and the discussion therein. 
global minimum point of $\ell_{N}(\boldsymbol{x})$ will be denoted by $\boldsymbol{\alpha}_{N *}$. We need the following differentiability assumption.

Assumption 4: Each of the functions $\left\{\mathbf{f}_{\mathbf{j}}(\mathbf{x})\right\}_{\dot{\mathbf{i}}=0}^{N-1}$ is at least twice differentiable with respect to $\mathbf{x}_{R}$ and $\mathbf{x}_{I}$ on $\mathcal{S}$.

Proposition 7: Let $\mathcal{H}_{N}\left(\boldsymbol{\alpha}_{N *}\right)$ be the Hessian matrix and $\mathrm{g}_{N}\left(\boldsymbol{\alpha}_{N *}\right)$ be the gradient vector (a row vector conventionally) of the loss function $\hat{\ell}_{N}(\boldsymbol{x})$ evaluated at $\boldsymbol{x}=\boldsymbol{\alpha}_{N *}$. Then, under Assumptions $1-4$, the asymptotic (as $N \rightarrow \infty$ ) estimation error $\tilde{\boldsymbol{\alpha}}_{N}:=\hat{\boldsymbol{\alpha}}_{N}-\boldsymbol{\alpha}_{N *}$ is given by

$$
\tilde{\boldsymbol{\alpha}}_{N}=-\mathcal{H}_{N}^{-1}\left(\boldsymbol{\alpha}_{N *}\right) \mathbf{g}_{N}^{\top}\left(\boldsymbol{\alpha}_{N *}\right) .
$$

Proof: Recall that $\mathrm{g}_{N}\left(\hat{\boldsymbol{\alpha}}_{N}\right)=\mathbf{0}_{1 \times 2 n}$. Using this fact in the Taylor's series expansion of $\hat{\ell}_{N}(\boldsymbol{x})$ in the neighborhood of $\boldsymbol{\alpha}_{N *}$ * and neglecting the third and higher order terms in $\hat{\boldsymbol{\alpha}}_{N}-\boldsymbol{\alpha}_{N *}$ (which is valid only asymptotically for large $N$, since $\hat{\boldsymbol{\alpha}}_{N}$ is a consistent estimator of $\boldsymbol{\alpha}_{N *}$ ) the proposition follows. The details of the proof is available in many related books and papers, see [12] for example.

Next, the properties of the Hessian matrix $\mathcal{H}_{N}\left(\boldsymbol{\alpha}_{N *}\right)$ and the gradient vector $\mathbf{g}_{N}\left(\boldsymbol{\alpha}_{N *}\right)$ will be explored. Since we are concerned about the asymptotic distribution of $\tilde{\boldsymbol{\alpha}}_{N}$, it is not required to establish the almost sure convergence of the relevant quantities [i.e., $\mathcal{H}_{N}\left(\boldsymbol{\alpha}_{N *}\right)$ and $\mathrm{g}_{N}\left(\boldsymbol{\alpha}_{N *}\right)$ ], but it would be sufficient to establish weak convergence in probability; see [23] and [24] for details. For what follows next, we use the following differentiation notations for a complex vector valued function $\mathbf{w}(\mathbf{a})$ of a real and vector valued parameter $\mathbf{a}=\left[\begin{array}{lll}a_{1} & \cdots & a_{m}\end{array}\right]^{\top}$, where we use

$$
\begin{aligned}
\mathbf{w}^{(\mathbf{k})}(\mathbf{a}) & :=\frac{\partial \mathbf{w}(\mathbf{a})}{\partial a_{\mathrm{k}}} \\
\mathbf{w}^{(\mathrm{kl})}(\mathbf{a}) & :=\frac{\partial^{2} \mathbf{w}(\mathbf{a})}{\partial a_{\mathrm{k}} \partial a_{l}}, \quad 1 \leq \mathrm{k}, \mathrm{I} \leq m .
\end{aligned}
$$

Further, the matrix of first-order derivatives will be denoted by

$$
\frac{\partial \mathbf{w}(\mathbf{a})}{\partial \mathbf{a}}:=\left[\mathbf{w}^{(1)}(\mathbf{a}) \quad \cdots \quad \mathbf{w}^{(m)}(\mathbf{a})\right]
$$

Assumption 5: The derivatives $\left\{\mathbf{f}_{\mathrm{k}}^{(\mathbf{i})}\right\}_{\mathfrak{i}=1}^{2 n}$ have bounded 2-norm for all $\mathrm{k}$ such that $0 \leq \mathrm{k}<N$. Similarly, the second derivatives $\left\{\mathbf{f}_{\mathbf{k}}^{(\mathrm{ij})}\right\}_{\mathrm{i}, \mathrm{j}=1}^{2 n}$ have bounded 2-norm for all $\mathbf{k}$ such that $0 \leq \mathrm{k}<N$.

Proposition 8: Let us define

$$
\mathbf{H}(\boldsymbol{x}):=\sum_{\mathbf{k}=0}^{N-1} \operatorname{Re}\left\{\left[\frac{\partial \mathbf{f}_{\mathbf{k}}(\boldsymbol{x})}{\partial \boldsymbol{x}}\right]^{*} \boldsymbol{\Sigma}_{\mathrm{k}}^{-1}\left[\frac{\partial \mathbf{f}_{\mathbf{k}}(\boldsymbol{x})}{\partial \boldsymbol{x}}\right]\right\} .
$$

Assume that the number of data sets $M>p+2$. Then under Assumptions 1, 4, and 5, $\mathbf{g}_{N}\left(\boldsymbol{\alpha}_{N *}\right)$ is Gaussian with mean zero and covariance matrix given by

$$
\mathbf{E} \mathbf{g}_{N}^{\top}\left(\boldsymbol{\alpha}_{N *}\right) \mathbf{g}_{N}\left(\boldsymbol{\alpha}_{N *}\right)=\frac{2(M-1)^{3}}{N^{2} M K\left(K^{2}-1\right)} \mathbf{H}\left(\boldsymbol{\alpha}_{N *}\right) .
$$

As a consequence, the estimation error $\tilde{\boldsymbol{\alpha}}_{N}$ is asymptotically $(N \rightarrow \infty)$ Gaussian.

Proof: Recall that $\boldsymbol{\alpha}_{N *}$ is the global minimum point of $\ell_{N}(\boldsymbol{x})$. Therefore

$$
\begin{aligned}
& \left.\frac{\partial \ell_{N}(\boldsymbol{x})}{\partial \boldsymbol{x}}\right|_{\boldsymbol{x}=\boldsymbol{\alpha}_{N *}}=0_{1 \times 2 n} \Rightarrow \\
& \sum_{\mathbf{k}=0}^{N-1} \operatorname{Re}\left\{\mathbf{f}_{\mathbf{k}}^{(\mathbf{i}) *}\left(\boldsymbol{\alpha}_{N *}\right) \boldsymbol{\Sigma}_{\mathbf{k}}^{-1} \mathbf{h}_{\mathbf{k}}\left(\boldsymbol{\alpha}_{N *}\right)\right\}=0
\end{aligned}
$$

for $\mathrm{i}=1, \ldots, 2 n$. Here, we have used (20) and (21) in the last equality. Differentiating $\lambda_{\mathbf{k}}(\boldsymbol{x})$ in (11) with respect to the ith element of $\boldsymbol{x}$ we get

$$
\begin{aligned}
& \left.\lambda_{\mathrm{k}}^{(\mathrm{i})}(\boldsymbol{x})\right|_{\boldsymbol{x}=\boldsymbol{\alpha}_{N *}}=-2 \operatorname{Re}\left\{\mathrm{f}_{\mathrm{k}}^{(\mathrm{i}) *}\left(\boldsymbol{\alpha}_{N *}\right) \hat{\boldsymbol{\Sigma}}_{\mathrm{k}}^{-1} \check{\mathbf{y}}_{\mathrm{k}}\right. \\
& \left.+\mathbf{f}_{\mathrm{k}}^{(\mathrm{i}) *}\left(\boldsymbol{\alpha}_{N *}\right) \boldsymbol{\Sigma}_{\mathrm{k}}^{-1} \mathbf{h}_{\mathrm{k}}\left(\boldsymbol{\alpha}_{N *}\right)\right\} .
\end{aligned}
$$

Hence, a typical element of the gradient vector $\mathbf{g}_{N}\left(\boldsymbol{\alpha}_{N *}\right)$ is given by

$$
\begin{aligned}
{\left[\mathbf{g}_{N}\left(\boldsymbol{\alpha}_{N *}\right)\right]_{\mathrm{i}} } & =\frac{1}{N} \sum_{\mathrm{k}=0}^{N-1} \lambda_{\mathrm{k}}^{(\mathrm{i})}\left(\boldsymbol{\alpha}_{N *}\right) \\
& =-\frac{2}{N} \sum_{\mathrm{k}=0}^{N-1} \operatorname{Re}\left\{\mathbf{f}_{\mathrm{k}}^{(\mathbf{i}) *}\left(\boldsymbol{\alpha}_{N *}\right) \hat{\boldsymbol{\Sigma}}_{\mathrm{k}}^{-1} \check{\mathbf{y}}_{\mathrm{k}}\right\}
\end{aligned}
$$

where we have used (43) in the last equality. Recall that $\lambda_{\mathbf{k}}(x)$ and $\lambda_{I}(\boldsymbol{x})$ are mutually independent if $\mathrm{k} \neq \mathrm{I}$. The same is true for their derivatives with respect to the elements of $x$. We will use the independence between $\check{\mathbf{y}}_{\mathbf{k}}$ and $\hat{\boldsymbol{\Sigma}}_{\mathrm{k}}$ and compute the expectation with respect to $\check{\mathbf{y}}_{k}$ first. By using circularity of $\check{\mathbf{y}}_{k}$, we get

$$
\begin{aligned}
\mathbf{E}_{\check{\mathbf{y}}_{\mathbf{k}}} & {\left[\mathbf{g}_{N}\left(\boldsymbol{\alpha}_{N *}\right)\right]_{\mathrm{i}}\left[\mathbf{g}_{N}\left(\boldsymbol{\alpha}_{N *}\right)\right]_{\mathbf{j}} } \\
& =\frac{2}{N^{2} M} \sum_{\mathbf{k}=0}^{N-1} \operatorname{Re}\left[\mathbf{f}_{\mathbf{k}}^{(\mathbf{i}) *}\left(\boldsymbol{\alpha}_{N *}\right) \hat{\boldsymbol{\Sigma}}_{\mathbf{k}}^{-1} \boldsymbol{\Sigma}_{\mathbf{k}} \hat{\boldsymbol{\Sigma}}_{\mathbf{k}}^{-1} \mathbf{f}_{\mathbf{k}}^{(\mathbf{j})}\left(\boldsymbol{\alpha}_{N *}\right)\right] .
\end{aligned}
$$

Next, we compute the expectation with respect to $\hat{\boldsymbol{\Sigma}}_{\mathrm{k}}$. We use Proposition 2 and second-order moment result (81) in the Appendix. We have

$$
\begin{aligned}
\mathbf{E}\left[\mathbf{g}_{N}\left(\boldsymbol{\alpha}_{N *}\right)\right]_{\mathrm{i}}\left[\mathrm{g}_{N}\left(\boldsymbol{\alpha}_{N *}\right)\right]_{\mathrm{j}}=\frac{2(M-1)^{3}}{N^{2} M K\left(K^{2}-1\right)} \\
\times \sum_{\mathbf{k}=0}^{N-1} \operatorname{Re}\left[\mathbf{f}_{\mathbf{k}}^{(\mathbf{i}) *}\left(\boldsymbol{\alpha}_{N *}\right) \boldsymbol{\Sigma}_{\mathbf{k}}^{-1} \mathbf{f}_{\mathbf{k}}^{(\mathbf{j})}\left(\boldsymbol{\alpha}_{N *}\right)\right] .
\end{aligned}
$$

From (46), we get (42) immediately. It is also easy to see from (44) that $\mathbf{g}_{N}\left(\boldsymbol{\alpha}_{N *}\right)$ is a zero mean random variable, which is a sum of independent Gaussian random variables with bounded second-order moments (by Assumption 5). Hence we can conclude that $\mathbf{g}_{N}\left(\boldsymbol{\alpha}_{N *}\right)$ is Gaussian. Since any linear transformation of a Gaussian random vector is Gaussian, it follows from Proposition 7, that the estimation error $\tilde{\boldsymbol{\alpha}}_{N}$ is asymptotically $(N \rightarrow \infty)$ Gaussian. 
Proposition 9: Under Assumptions 1, 4, and 5, the Hessian matrix $\mathcal{H}_{N}\left(\boldsymbol{\alpha}_{N *}\right)$ converges in mean squares sense as $N \rightarrow \infty$ to its expected value $\mathbf{E} \mathcal{H}_{N}\left(\boldsymbol{\alpha}_{N *}\right)$ given by

$$
\mathbf{E} \mathcal{H}_{N}\left(\boldsymbol{\alpha}_{N *}\right)=\frac{2(M-1)}{N K}\left\{\mathbf{H}\left(\boldsymbol{\alpha}_{N *}\right)-\mathbf{H}_{1}\left(\boldsymbol{\alpha}_{N *}\right)\right\}
$$

where $\mathbf{H}(\boldsymbol{x})$ is defined in (41) and $\mathbf{H}_{1}(\boldsymbol{x})$ is given element-wise as

$$
\left[\mathbf{H}_{1}(\boldsymbol{x})\right]_{\mathrm{ij}}=\sum_{\mathrm{k}=0}^{N-1} \operatorname{Re}\left\{\mathrm{f}_{\mathrm{k}}^{(\mathrm{ij}) *}(\boldsymbol{x}) \hat{\boldsymbol{\Sigma}}_{\mathrm{k}}^{-1} \mathbf{h}_{\mathrm{k}}(\boldsymbol{x})\right\} .
$$

Proof: Consider a typical element of the Hessian matrix $\mathcal{H}_{N}\left(\boldsymbol{\alpha}_{N *}\right)$ :

$$
\left.\hat{\ell}_{N}^{(i \mathbf{j})}(\boldsymbol{x})\right|_{\boldsymbol{x}=\boldsymbol{\alpha}_{N *}}=\left.\frac{1}{N} \sum_{\mathrm{k}=0}^{N-1} \lambda_{\mathrm{k}}^{(\mathrm{ij})}(\boldsymbol{x})\right|_{\boldsymbol{x}=\boldsymbol{\alpha}_{N *}} .
$$

Differentiating (11) twice with respect to $\boldsymbol{x}_{\mathrm{i}}$ and $\boldsymbol{x}_{\mathrm{j}}$, respectively, we have

$$
\begin{aligned}
\lambda_{\mathrm{k}}^{(\mathrm{ij})}(\boldsymbol{x})=2 \operatorname{Re} & {\left[\mathrm{f}_{\mathrm{k}}^{(\mathrm{i}) *}(\boldsymbol{x}) \hat{\Sigma}_{\mathrm{k}}^{-1} \mathbf{f}_{\mathrm{k}}^{(\mathrm{j})}(\boldsymbol{x})\right.} \\
& \left.-\mathbf{f}_{\mathrm{k}}^{(\mathrm{ij}) *}(\boldsymbol{x}) \hat{\Sigma}_{\mathrm{k}}^{-1} \breve{\mathbf{y}}-\mathbf{f}_{\mathrm{k}}^{(\mathrm{ij}) *}(\boldsymbol{x}) \hat{\Sigma}_{\mathrm{k}}^{-1} \mathbf{h}_{\mathbf{k}}(\boldsymbol{x})\right] .
\end{aligned}
$$

It is readily verified using (24) and (26) that

$$
\begin{aligned}
\mathbf{E} \lambda_{\mathrm{k}}^{(\mathrm{ij})}(\boldsymbol{x})=\frac{2(M-1)}{K} \operatorname{Re}\left[\mathbf{f}_{\mathrm{k}}^{(\mathbf{i}) *}(\boldsymbol{x}) \boldsymbol{\Sigma}_{\mathrm{k}}^{-1} \mathbf{f}_{\mathrm{k}}^{(\mathrm{j})}(\boldsymbol{x})\right. \\
\left.-\mathbf{f}_{\mathrm{k}}^{(\mathrm{ij}) *}(\boldsymbol{x}) \boldsymbol{\Sigma}_{\mathrm{k}}^{-1} \mathbf{h}_{\mathrm{k}}(\boldsymbol{x})\right] .
\end{aligned}
$$

Now, it is straightforward to deduce (47) from (49) and (51). It remains to show that every element of $\mathcal{H}_{N}(\boldsymbol{\alpha})$ converges in mean square sense as $N \rightarrow \infty$ to its expected value. Aiming this it is sufficient to show that $\operatorname{Var}\left(\lambda_{\mathrm{k}}^{(\mathrm{ij})}\right)$ is bounded for all $\mathrm{i}, \mathrm{j}$, and $\mathrm{k}$. We will explore the second-order moment of $\lambda_{\mathrm{k}}^{(\mathrm{ij})}$ in that goal. Evaluating the expectation with respect to $\breve{\mathbf{y}}_{\mathrm{k}}$ first, we get

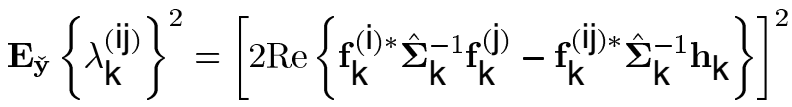

$$
\begin{aligned}
& +\frac{2}{M} \mathbf{f}_{\mathrm{k}}^{(\mathrm{ij}) *} \hat{\boldsymbol{\Sigma}}_{\mathrm{k}}^{-1} \boldsymbol{\Sigma}_{\mathrm{k}} \hat{\Sigma}_{\mathrm{k}}^{-1} \mathbf{f}_{\mathrm{k}}^{(\mathrm{ij})}
\end{aligned}
$$

Using the second-order moment results given in the Appendix, it is now easy to verify under Assumption 5 that the second-order moment of $\lambda_{\mathrm{k}}^{(\mathrm{ij})}(\boldsymbol{\alpha})$ is bounded for all $\mathrm{i}, \mathrm{j}$, and $\mathrm{k}$. With this, the proposition is proved.

Note that if the model (2) is correct then $\boldsymbol{\alpha}_{N *}=\boldsymbol{\alpha}$ and $\mathbf{h}_{\mathbf{k}}(\boldsymbol{\alpha})=0$ for all $\mathbf{k}$. Therefore, in such cases

$$
\mathbf{H}_{1}(\boldsymbol{\alpha})=0_{2 n \times 2 n} \Rightarrow \mathbf{E} \mathcal{H}_{N}(\boldsymbol{\alpha})=\frac{2(M-1)}{N K} \mathbf{H}(\boldsymbol{\alpha}) .
$$

This is a key observation in the proof of the following main result of this section.
Proposition 10: Assume that the model (2) is correct and Assumptions 1-5 hold. Then, if the number of data sets $M>p+2$ the asymptotic $(N \rightarrow \infty)$ covariance matrix of the estimated parameter vector $\hat{\boldsymbol{\alpha}}_{N}$ is given by

$$
\mathbf{C}_{\boldsymbol{\alpha}}=\frac{\delta}{2 M} \mathbf{H}^{-1}(\boldsymbol{\alpha}) \quad \delta=\frac{(M-1)(M-p-1)}{(M-p)(M-p-2)} .
$$

Proof: The proof will follow from Propositions 7-9. First, we can approximate

$$
\mathcal{H}_{N}(\boldsymbol{\alpha}) \approx \mathbf{E} \mathcal{H}_{N}(\boldsymbol{\alpha})
$$

for large $N$ by Proposition 9 . Therefore, by Proposition 7, the estimation error can be written for large $N$ as

$$
\tilde{\boldsymbol{\alpha}}_{N}=-\left\{\mathbf{E} \mathcal{H}_{N}(\boldsymbol{\alpha})\right\}^{-1} \mathbf{g}_{N}^{\top}(\boldsymbol{\alpha}) .
$$

It is now straightforward to see that the asymptotic covariance matrix of the parameter estimates is given by

$$
\begin{aligned}
\mathbf{E} \tilde{\boldsymbol{\alpha}}_{N} \tilde{\boldsymbol{\alpha}}_{N}^{\top}=\left\{\mathbf{E} \mathcal{H}_{N}(\boldsymbol{\alpha})\right\}^{-1} \mathbf{E} \\
\\
\left\{\mathbf{g}_{N}^{\top}(\boldsymbol{\alpha}) \mathbf{g}_{N}(\boldsymbol{\alpha})\right\}\left\{\mathbf{E} \mathcal{H}_{N}(\boldsymbol{\alpha})\right\}^{-1}
\end{aligned}
$$

which leads to (54) by using (42) and (47).

The asymptotic covariance matrix achieved by the maximum likelihood estimate is given by $[2 M \mathbf{H}(\boldsymbol{\alpha})]^{-1}$. This is also the Cramér-Rao bound for the estimation problem. Therefore, the loss of efficiency for using the nonparametric noise model is quantified by the factor $\delta$. Note that $\delta>1$. It is also readily verified that

$$
\delta=1+\frac{p}{M}+O\left(M^{-2}\right) \Rightarrow \lim _{M \rightarrow \infty} \delta=1 .
$$

If the observed data are scalar valued, i.e., $p=1$

$$
\delta=\frac{M-2}{M-3} .
$$

The same result was reported in [14] in context of identification of SISO dynamic errors-in-variables (EIV) models using nonparametric noise models estimated from limited number of data sets. Note that for $p=1$, the consistency and accuracy results are valid for $M>3$. Comparing with the results reported in [14], the requirement for consistency is the same. However, the requirement for the asymptotic covariance to exist is less stringent $^{6}$ ( $M \geq 6$ as required by the SISO dynamic errors-in-variables models).

Assumption 6: If $\mathbf{x} \in \mathcal{S}$, the functions $\left\{\mathbf{f}_{\mathbf{k}}(\mathbf{x})\right\}_{\mathrm{k}=0}^{N-1}$ are analytic functions of the complex valued parameter vector $\mathbf{x}$.

${ }^{6}$ The minimal number of independent experiments $M$ between the output error model case and the EIV case differs due to the fact that the residual covariance matrix in the EIV case is $\boldsymbol{\theta}$-dependent. The expression for the covariance matrix of the parameters will also be a function of the derivative of the inverse of the residual covariance matrix. The existence of the second order moments of the latter will increase the minimal value of $M$. 
Proposition 11: Under Assumptions 1-6, the complex valued parameter estimate $\hat{\boldsymbol{\theta}}_{N}$ is asymptotically (as $N \rightarrow \infty$ ) circular with covariance matrix $\mathbf{C}_{\boldsymbol{\theta}}$, i.e.,

$$
\begin{aligned}
& \mathbf{E}\left(\hat{\boldsymbol{\theta}}_{N}-\boldsymbol{\theta}\right)\left(\hat{\boldsymbol{\theta}}_{N}-\boldsymbol{\theta}\right)^{*}=\mathbf{C}_{\boldsymbol{\theta}} \\
& \mathbf{E}\left(\hat{\boldsymbol{\theta}}_{N}-\boldsymbol{\theta}\right)\left(\hat{\boldsymbol{\theta}}_{N}-\boldsymbol{\theta}\right)^{\top}=0_{n \times n}
\end{aligned}
$$

where

$$
\begin{aligned}
& \mathbf{C}_{\boldsymbol{\theta}}=\frac{\delta}{M}\left\{\sum_{\mathbf{k}=0}^{N-1} \boldsymbol{\Lambda}_{\mathbf{k}}\right\}^{-1} \\
& \boldsymbol{\Lambda}_{\mathbf{k}}=\left[\frac{\partial \mathbf{f}_{\mathbf{k}}(\boldsymbol{\alpha})}{\partial \mathbf{x}_{R}}\right]^{*} \boldsymbol{\Sigma}_{\mathbf{k}}^{-1}\left[\frac{\partial \mathbf{f}_{\mathbf{k}}(\boldsymbol{\alpha})}{\partial \mathbf{x}_{R}}\right] .
\end{aligned}
$$

Proof: By Cauchy-Riemann's conditions for analytic functions, we know that

$$
\frac{\partial \mathbf{f}_{\mathbf{k}}(\boldsymbol{\alpha})}{\partial \boldsymbol{x}}=\left[\begin{array}{ll}
\frac{\partial \mathbf{f}_{\mathbf{K}}(\boldsymbol{\alpha})}{\partial \mathbf{x}_{R}} & \mathrm{i} \frac{\partial \mathbf{f}_{\mathbf{k}}(\boldsymbol{\alpha})}{\partial \mathbf{x}_{R}}
\end{array}\right] .
$$

Therefore, by straightforward matrix manipulation, we get

$$
\left[\frac{\partial \mathbf{f}_{\mathrm{k}}(\boldsymbol{\alpha})}{\partial \boldsymbol{x}}\right]^{*} \boldsymbol{\Sigma}_{\mathrm{k}}^{-1}\left[\frac{\partial \mathbf{f}_{\mathrm{k}}(\boldsymbol{\alpha})}{\partial \boldsymbol{x}}\right]=\left[\begin{array}{cc}
\boldsymbol{\Lambda}_{\mathrm{k}} & \mathrm{i} \boldsymbol{\Lambda}_{\mathrm{k}} \\
-\mathrm{i} \boldsymbol{\Lambda}_{\mathrm{k}} & \boldsymbol{\Lambda}_{\mathrm{k}}
\end{array}\right]
$$

Now, combining (41), (54), and (61), we get

$$
\begin{aligned}
\mathbf{C}_{\boldsymbol{\alpha}} & =\left[\frac{2 M}{\delta} \mathbf{H}(\boldsymbol{\alpha})\right]^{-1} \\
& =\left[\begin{array}{cc}
\operatorname{Re}\left\{\mathbf{C}_{\boldsymbol{\theta}} / 2\right\}^{-1} & -\operatorname{Im}\left\{\mathbf{C}_{\boldsymbol{\theta}} / 2\right\}^{-1} \\
\operatorname{Im}\left\{\mathbf{C}_{\boldsymbol{\theta}} / 2\right\}^{-1} & \operatorname{Re}\left\{\mathbf{C}_{\boldsymbol{\theta}} / 2\right\}^{-1}
\end{array}\right]^{-1} \\
& =\left[\begin{array}{cc}
\operatorname{Re}\left\{\mathbf{C}_{\boldsymbol{\theta}} / 2\right\} & -\operatorname{Im}\left\{\mathbf{C}_{\boldsymbol{\theta}} / 2\right\} \\
\operatorname{Im}\left\{\mathbf{C}_{\boldsymbol{\theta}} / 2\right\} & \operatorname{Re}\left\{\mathbf{C}_{\boldsymbol{\theta}} / 2\right\}
\end{array}\right]
\end{aligned}
$$

where in the last equality, we have used well-known isomorphism results, see [18]. From (64), it is easy to verify (60). With this, the proposition follows.

\section{Loss Function Properties at the Global Minimum}

The minimum value of the loss function often gives us the indications about the validity of the model structure after the identification process. In many identification problems, we can predict the minimum value of the cost function approximately, in the sense that the mean value and the variance of the loss function at its global minimum is known to us a priori, provided there is no modeling error. In such circumstances if the minimum value of the cost function differs significantly from its predicted value, the user gets an indication that the model structure used in the identification process may not be enough to describe the data. In order to be able to compute these statistics of the loss function at the global minimum, we often need to know the noise statistics. Using the nonparametric noise model, it is therefore possible to compute the statistics of the cost function at its global minimum. In this section we shall compute the mean and approximate variance of the minimum value of the cost function. Here, again we shall not assume (unless mentioned otherwise) that model (2) is correct. The following proposition is concerning the mean value.
Proposition 12: Under Assumptions 1-5, as $N \rightarrow \infty$ the expected minimum value of the loss function $\hat{\ell}_{N}(\mathbf{x})$ is given by

$$
\mathbf{E} \hat{\ell}_{N}\left(\hat{\boldsymbol{\alpha}}_{N}\right)=\frac{M-1}{K N} \sum_{\mathbf{i}=0}^{N-1}\left[\mathbf{h}_{\mathbf{i}}^{*}\left(\boldsymbol{\alpha}_{N *}\right) \boldsymbol{\Sigma}_{\mathbf{i}}^{-1} \mathbf{h}_{\mathbf{i}}\left(\boldsymbol{\alpha}_{N *}\right)+\frac{p}{M}\right] .
$$

Proof: Expanding the loss function $\hat{\ell}_{N}(\boldsymbol{x})$ in Taylor's series in the neighborhood of $\boldsymbol{\alpha}_{N *}$ and neglecting higher order terms in $\tilde{\boldsymbol{\alpha}}_{N}$ (which is valid only for large $N$ ) we get

$$
\begin{aligned}
\hat{\ell}_{N}\left(\hat{\boldsymbol{\alpha}}_{N}\right) \\
=\hat{\ell}_{N}\left(\boldsymbol{\alpha}_{N *}\right)+\mathbf{g}_{N}\left(\boldsymbol{\alpha}_{N *}\right) \tilde{\boldsymbol{\alpha}}_{N}+\frac{1}{2} \tilde{\boldsymbol{\alpha}}_{N}^{\top} \mathcal{H}_{N}\left(\boldsymbol{\alpha}_{N *}\right) \tilde{\boldsymbol{\alpha}}_{N} \\
=\hat{\ell}_{N}\left(\boldsymbol{\alpha}_{N *}\right)-\frac{1}{2} \mathbf{g}_{N}\left(\boldsymbol{\alpha}_{N *}\right) \mathcal{H}_{N}^{-1}\left(\boldsymbol{\alpha}_{N *}\right) \mathbf{g}_{N}^{\top}\left(\boldsymbol{\alpha}_{N *}\right) \\
\approx \hat{\ell}_{N}\left(\boldsymbol{\alpha}_{N *}\right)-\frac{N K}{4(M-1)} \\
\quad \times \mathbf{g}_{N}\left(\boldsymbol{\alpha}_{N *}\right)\left\{\mathbf{H}\left(\boldsymbol{\alpha}_{N *}\right)-\mathbf{H}_{1}\left(\boldsymbol{\alpha}_{N *}\right)\right\}^{-1} \mathbf{g}_{N}^{\top}\left(\boldsymbol{\alpha}_{N *}\right)
\end{aligned}
$$

where the second equality uses (38) and the last equality follows using Proposition 9. Taking expectation and using (42) gives

$$
\begin{aligned}
\mathbf{E} \hat{\ell}_{N}\left(\hat{\boldsymbol{\alpha}}_{N}\right)= & \ell_{N}\left(\hat{\boldsymbol{\alpha}}_{N *}\right)-\frac{(M-1)^{2}}{2 N M\left(K^{2}-1\right)} \\
& \times \operatorname{Tr}\left[\left\{\mathbf{H}\left(\boldsymbol{\alpha}_{N *}\right)-\mathbf{H}_{1}\left(\boldsymbol{\alpha}_{N *}\right)\right\}^{-1} \mathbf{H}\left(\boldsymbol{\alpha}_{N *}\right)\right] .
\end{aligned}
$$

Whenever $M \ll N$, the aforementioned second term can be neglected, compared to the first term which is bounded. ${ }^{7}$ Therefore, using Proposition 3 we get (65).

The first term in (65) is the contribution due to the model error, while the second term corresponds to the noise contribution. Note that in absence of any model error we have $\boldsymbol{\alpha}_{N *}=\boldsymbol{\alpha}$ and $\mathbf{h}_{\mathbf{k}}(\boldsymbol{\alpha})=0_{p \times 1}$. Therefore

$$
\mathbf{E} \hat{\ell}_{N}(\boldsymbol{\alpha}) \approx \frac{(M-1) p}{K M}=\frac{(M-1) p}{(M-p-1) M} .
$$

If we take $p=1$, this is in perfect accordance with the results obtained in [14]. Next, we compute the variance of the cost function at the global minimum point. It is reasonable to approximate

$$
\operatorname{Var}\left[\hat{\ell}_{N}\left(\hat{\boldsymbol{\alpha}}_{N}\right)\right]=\operatorname{Var}\left[\hat{\ell}_{N}\left(\boldsymbol{\alpha}_{N *}\right)\right]
$$

This can be computed easily using (27) and (31). Here, we consider the case where there is no modeling error. Using $\mathbf{h}_{\mathbf{k}}(\boldsymbol{\alpha})=$ $0_{p \times 1}$ in (27) and (31) we see that

$$
\operatorname{Var}\left[\hat{\ell}_{N}\left(\hat{\boldsymbol{\alpha}}_{N}\right)\right]=\frac{p(M-1)^{3}}{N M^{2}(M-p-1)^{2}(M-p-2)} .
$$

Putting $p=1$, this again is in perfect accordance with the results obtained in [14].

${ }^{7}$ Note that we cannot neglect the second term if $M$ and $N$ are of the same order. However, such a situation is rare in practice 
TABLE I

Simulation RESUlts Form 1000 MONTE CARLO Simulations

\begin{tabular}{|c|c|c|c|c|}
\hline Parameter & $a_{1}$ & $a_{2}$ & $b_{1}$ & $b_{2}$ \\
\hline \hline True Value & 0.5 & -0.9 & -2.0 & 0.1 \\
\hline Mean & 0.4997 & -0.9000 & -1.9998 & 0.0994 \\
\hline $\begin{array}{c}\text { M.S.E } \times 10^{4} \\
\text { (numerical) }\end{array}$ & 0.316 & 0.016 & 1.428 & 1.602 \\
\hline $\begin{array}{c}\text { M.S.E } \times 10^{4} \\
\text { (analytical) }\end{array}$ & 0.312 & 0.018 & 1.500 & 1.634 \\
\hline
\end{tabular}

\section{SimULATION EXAMPLE}

In this section, we consider the parametric estimation of a multidimensional transfer function of the form

$$
\mathbf{G}\left(q^{-1}, \boldsymbol{\theta}\right)=\frac{1}{\left(1+a_{1} q^{-1}\right)\left(1+a_{2} q^{-1}\right)}\left[\begin{array}{l}
1+b_{1} q^{-1} \\
1+b_{2} q^{-1}
\end{array}\right]
$$

using the measurements of the frequency response functions at $N=512$ discrete frequency points. The problem here is to estimate the parameter vector

$$
\boldsymbol{\theta}=\left[\begin{array}{llll}
a_{1} & a_{2} & b_{1} & b_{2}
\end{array}\right]^{\top} .
$$

Similar approach is very common in system identification. See [8] for a typical practical application. However, the example here is quite simplified. In practical problems the dimension of the transfer function can be quite high. In the simulations, the value of the parameters were taken as $a_{1}=0.9, a_{2}=-0.5$, $b_{1}=2, b_{2}=0.1$. The measurements were corrupted by complex Gaussian measurement noise, where the covariance matrix of the noise at frequency $\omega$ was set to

$$
\boldsymbol{\Psi}(\omega)=\left[\begin{array}{cc}
1.02+0.2 \cos \omega & -0.01+0.1 \mathrm{e}^{-\mathrm{i} \omega} \\
-0.01+0.1 \mathrm{e}^{\mathrm{i} \omega} & 1.01-0.2 \cos \omega
\end{array}\right] .
$$

In terms of the notations used in this paper, we have

$$
\mathbf{f}_{\mathrm{k}}(\boldsymbol{\theta})=\mathbf{G}\left(\mathrm{e}^{-\mathrm{i} \omega} \mathrm{k}, \boldsymbol{\theta}\right), \boldsymbol{\Sigma}_{\mathrm{k}}=\boldsymbol{\Psi}\left(\omega_{\mathrm{k}}\right)
$$

In the simulation we took $M=6$. Note that in this case the dimension of the measurement vector is $p=2$. Therefore, the minimum value of $M$ required to ensure the consistency of the estimates is 5. In Table I we have summarized the results obtained from the simulations. The results are obtained from 1000 Monte-Carlo simulations. In each simulation the number of data points $N$ was taken as 512. In Table I, we have listed the true value, mean from the simulations, the mean square error (M.S.E) from the simulation and the analytical mean square error for each parameter. The analytical M.S.E is computed using Proposition 10. As we see in Table I that the estimates are practically unbiased and the numerical M.S.E is very well predicted by the analytical M.S.E. As predicted by (68), the mean value of the loss function at its global minimum in this case is $(2 \times 5) /(6 \times 3)=0.5556$. The corresponding value from the numerical simulations was 0.5600 . The variance of the loss function at its global minimum in this case, see (70), is $\left(2 \times 5^{3}\right) /\left(512 \times 6^{2} \times 3^{2} \times 2\right)=7.535 \times 10^{-4}$, while the corresponding quantity from the numerical simulations was found to be $7.408 \times 10^{-4}$. These observations confirm the validity of the analytical results presented in Section III.

\section{CONCLUSION}

In this paper, we have addressed the problem of fitting multidimensional parametric models in the frequency domain using nonparametric noise models. In order to obtain the nonparametric estimate of the noise covariance matrix, we need multiple measurements of the data at every frequency point. This can be achieved by employing periodic inputs, where the measurement is carried out for $M$ periods. A nonparametric estimate of the noise statistics is obtained from $M$ measurements corresponding to $M$ periods. The estimated noise model is then substituted for the the true noise covariance matrix in the maximum likelihood loss function to obtain suboptimal parameter estimates. Here, we have presented an analysis of the resulting estimates. We have derived the conditions for consistency. It is required to have $M>p+1$, where $p$ is the dimension of the measurements, to ensure consistency of the estimates. We have also derived the asymptotic (as number of data points $N \rightarrow \infty$ ) covariance matrix of the estimates and quantified the relative asymptotic efficiency of the estimates. It turns out that for the second order statistics of the parameter estimates to exist we need $M>p+2$. The resulting covariance matrix is proportional to that of the maximum-likelihood estimate. The proportionality constant $\delta>1$ depends on $M$, see (54). Also, as $M \rightarrow \infty$, we have $\delta \rightarrow 1$, as expected. Next, we have derived the first- and second-order statistics of the cost function at the global minimum point, which can be used for model validation. When $M \ll N$, both the mean and the variance of the cost function at the minimum point depend only on, and $p$. This gives us a handy tool to detect the model errors. The results presented here are in agreement with the results presented in [14], where parametric identification of SISO dynamic errors-in-variables model was considered (in this case $p=1$ ). We have illustrated the result using numerical simulations. Results therefrom confirm the validity of the analytical findings.

\section{APPENDIX A}

In this Appendix, we state and prove a few second-order moment results relating the complex inverse Wishart distribution. These results are required to prove the convergence results presented in this paper. Here, we will denote the matrices $\boldsymbol{\Sigma}, \boldsymbol{\Sigma}^{-1}$, and $\hat{\boldsymbol{\Sigma}}^{-1}$ element-wise as as

$$
\Sigma=\left(\sigma_{\mathrm{ij}}\right), \Sigma^{-1}=\left(\varsigma_{\mathrm{ij}}\right) \hat{\Sigma}^{-1}=\left(\hat{\varsigma}_{\mathrm{ij}}\right) .
$$

From (18), we know that

$$
\mathbf{E} \hat{\mathrm{s}}_{\mathrm{ij}} \hat{\mathrm{s}}_{\mathrm{kl}}=\frac{(M-1)^{2}}{K^{2}-1}\left[\varsigma_{\mathrm{ij}} \varsigma_{\mathrm{kl}}+\frac{1}{K} \varsigma_{\mathrm{kj}} \varsigma_{\mathrm{il}}\right] .
$$


Let us introduce $p \times 1$ deterministic vectors $\mathbf{h}, \mathbf{g}, \mathbf{f}$ and $\mathbf{e}$ given element-wise as

$$
\mathbf{h}=\left(h_{\mathbf{i}}\right) \quad \mathbf{f}=\left(f_{\mathbf{i}}\right) \quad \mathbf{g}=\left(g_{\mathbf{i}}\right) \quad \mathbf{e}=\left(e_{\mathbf{i}}\right) .
$$

Then, using (76), we get the following moment results:

$$
\begin{aligned}
& \mathbf{E}\left\{\mathbf{e}^{*} \hat{\boldsymbol{\Sigma}}^{-1} \mathbf{f} \mathrm{g}^{*} \hat{\boldsymbol{\Sigma}}^{-1} \mathbf{h}\right\} \\
& =\sum_{\mathrm{i}, \mathrm{j}, \mathrm{k}, \mathrm{I}} \mathbf{E} e_{\mathrm{i}}^{*} \hat{\mathrm{s}}_{\mathrm{ij}} f_{\mathrm{j}} g_{\mathbf{k}}^{*} \hat{\mathrm{s}}_{\mathrm{kl}} h_{\mathrm{I}} \\
& =\frac{(M-1)^{2}}{K^{2}-1}\left\{\mathbf{e}^{*} \boldsymbol{\Sigma}^{-1} \mathbf{f} \mathbf{g}^{*} \boldsymbol{\Sigma}^{-1} \mathbf{h}\right. \\
& \left.+\frac{1}{K} \mathbf{e}^{*} \boldsymbol{\Sigma}^{-1} \mathbf{h} \mathbf{g}^{*} \boldsymbol{\Sigma}^{-1} \mathbf{f}\right\} \\
& \mathbf{E} \operatorname{Tr}\left\{\hat{\boldsymbol{\Sigma}}^{-1} \boldsymbol{\Sigma} \hat{\boldsymbol{\Sigma}}^{-1} \boldsymbol{\Sigma}\right\} \\
& =\sum_{\mathrm{i}, \mathrm{j}, \mathrm{k}, \mathrm{l}} \mathbf{E} \hat{\mathrm{sij}}_{\mathrm{j}} \sigma_{\mathrm{jk}} \hat{\varsigma}_{\mathrm{k} \mathbf{l}} \sigma_{\mathrm{li}} \\
& =\frac{(M-1)^{2}}{K^{2}-1}\left[p+\frac{p^{2}}{K}\right] \\
& \mathbf{E} \operatorname{Tr}^{2}\left\{\hat{\boldsymbol{\Sigma}}^{-1} \boldsymbol{\Sigma}\right\} \\
& =\sum_{\mathrm{i}, \mathrm{j}, \mathrm{k}, \mathrm{I}} \mathbf{E} \hat{\varsigma}_{\mathrm{ij}} \sigma_{\mathrm{ji}} \hat{\varsigma}_{\mathbf{k} \mid} \sigma_{\mathrm{Ik}} \\
& =\frac{(M-1)^{2}}{K^{2}-1}\left[p^{2}+\frac{p}{K}\right] \\
& \mathbf{E}\left\{\mathbf{h}^{*} \hat{\boldsymbol{\Sigma}}^{-1} \boldsymbol{\Sigma} \hat{\boldsymbol{\Sigma}}^{-1} \mathbf{f}\right\} \\
& =\sum_{\mathrm{i}, \mathrm{j}, \mathrm{k}, \mathrm{I}} \mathbf{E} h_{\mathrm{i}}^{*} \hat{\mathrm{s}}_{\mathrm{ij}} \sigma_{\mathrm{jk}} \hat{\mathrm{s}}_{\mathrm{k} l} f_{\mathrm{I}} \\
& =\frac{(M-1)^{2}}{K^{2}-1}\left[1+\frac{p}{K}\right] \mathbf{h}^{*} \boldsymbol{\Sigma}^{-1} \mathbf{f} \\
& \mathbf{E}\left[\mathbf{h}^{*} \hat{\boldsymbol{\Sigma}}^{-1} \mathbf{h} \operatorname{Tr}\left\{\hat{\boldsymbol{\Sigma}}^{-1} \mathbf{\Sigma}\right\}\right] \\
& =\sum_{\mathrm{i}, \mathrm{j}, \mathrm{k}, \mathrm{I}} \mathbf{E} h_{\mathrm{i}}^{*} \hat{\mathrm{s}}_{\mathrm{ij}} h_{\mathrm{j}} \sigma_{\mathrm{Ik}} \hat{\varsigma}_{\mathrm{kl}} \\
& =\frac{(M-1)^{2}}{K^{2}-1}\left[p+\frac{1}{K}\right] \mathbf{h}^{*} \boldsymbol{\Sigma}^{-1} \mathbf{h} .
\end{aligned}
$$

[5] J. Schoukens, P. Guillaume, and R. Pintelon, , K. Godfrey, Ed., "Design of broadband excitation signals," in Perturbation Signals for System Identification. Englewood Cliffs, NJ: Prentice-Hall, 1993, pp. $126-159$.

[6] R. Pintelon and J. Schoukens, System Identification: A Frequency Domain Approach. New York: IEEE Press, 2001.

[7] C. P. Coelho, J. R. Phillips, and L. M. Silveira, "Optimization based passive constrained fitting," in Proc. ICCAD, Nov. 2002, pp. 775-780.

[8] R. A. Callafon, D. de Roover, and P. M. J. Van den Hof, "Multivariable least squares frequency domain identification using polynomial matrix fraction descriptions," in Proc. 35th Int. Conf. Decision and Control, Kobe, Japan, Dec. 1996, pp. 2030-2035.

[9] D. K. de Vries and P. M. J. V. den Hof, "Frequency domain identification with generalized orthonormal basis functions," IEEE Trans. Autom. Control, vol. 43, no. 5, pp. 656-669, May 1998.

[10] D. S. Bayard, "High order multivariable transfer function curve fitting: Algorithms, sparse matrix methods and experimental results," Automatica, vol. 30, no. 9, pp. 1439-1444, 1994.

[11] G. Gu and P. P. Khargonekar, "A class of algorithms for identification in $H_{\infty}$, , Automatica, vol. 28, pp. 299-312, 1992.

[12] T. Söderström and P. Stoica, System Identification.. Hemel Hempstead, U.K.: Prentice-Hall, 1989.

[13] L. Ljung, System Identification-Theory for the User, 2nd ed. Upper Saddle River, NJ: Prentice-Hall, 1999.

[14] J. Schoukens, R. Pintelon, G. Vandersteen, and P. Guillaume, "Frequency-domain system identification using nonparametric noise models estimated from a small number of data sets," Automatica, vol. 33:6, pp. 1073-1086, Jun. 1997.

[15] R. Pintelon, "Frequency-domain subspace system identification using nonparametric noise models," Automatica, vol. 38, no. 8, pp. 1295-1311, Aug. 2002.

[16] R. Pintelon, J. Schoukens, and Y. Rolain, "Uncertainty of transfer function modelling using prior estimated noise models," Automatica, vol. 39, no. 10, pp. 1721-1733, Oct. 2003.

[17] D. R. Brillinger, Time Series: Data Analysis and Theory. New York: McGraw-Hill, 1981.

[18] N. R. Goodman, "Statistical analysis based on certain multivariate complex distribution (an introduction)," Ann. Math. Statist., vol. 34:1, pp. 152-177, 1963

[19] T. W. Anderson, An Introduction to Multivariate Statistical Analysis. New York: Wiley, 2003.

[20] D. Maiwald and D. Kraus, "Calculation of moments of complex wishart and complex inverse wishart distributed matrices," in Proc. Inst. Elect. Eng. Radar, Sonar Navigation, Aug. 2000, vol. 147, no. 4, pp. 162-168.

[21] D. von Rosen, "Moments of the inverted wishart distribution," Scandinavian J. Statist., vol. 15, pp. 97-109, 1988.

[22] A. Papoulis, Probability, Random Variables, and Stochastic Processes. New York: McGraw-Hill, 1991.

[23] P. Billingsley, Probability and Measure, 3rd ed. New York: Wiley, 1995.

[24] R. G. Laha and V. K. Rohatgi, Probability Theory. New York: Wiley, 1979.

[25] P. Janssen and P. Stoica, "On the expectation of the product of four matrix-valued Gaussian random variables," IEEE Trans. Autom. Control, vol. AC-33, no. 9, pp. 867-870, Sep. 1988.

[26] B. Porat, Digital Processing of Random Signals. Englewood Cliffs, NJ: Prentice-Hall, 1994.

[27] T. Söderström, "Convergence properties of the generalized least squares identification algorithm," Automatica, vol. 10, pp. 617-626, 1974.

\section{REFERENCES}

[1] R. Hilfer, "Fitting the excess wing in the dielectric $\alpha$-relaxation of propylene carbonate," J. Phys.: Condensed Matter, vol. 14, pp. 2297-2301, 2002.

[2] J. Slotboom, C. Boesch, and R. Kreis, "Versatile frequency domain fitting using time domain models and prior knowledge," Magn. Reson. Med., vol. 39, pp. 899-911, Jun. 1998.

[3] S. Grivet-Talocia, I. S. Stievano, I. A. Maio, and F. Canavero, "Time-domain and frequency-domain macromodeling: Application to package structures," in Proc. IEEE Int. Symp. EMC, Boston, MA, Aug. 2003, pp. 570-574.

[4] L. Hillström, M. Mossberg, and B. Lundberg, "Identification of complex modulus from measured strains on an axially impacted bar using least squares," J. Sound Vibrat., vol. 230, pp. 689-707, 2000.

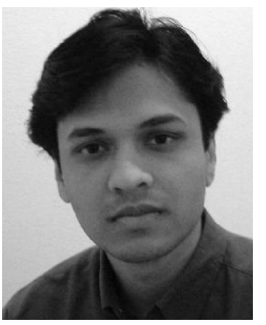

Kaushik Mahata (M'04) received the M.E. degree in signal processing from the Indian Institute of Science, Bangalore, India, in 2000, and the Ph.D. degree in signal processing from Uppsala University, Uppsala, Sweden, in 2003.

Currently, he is a Research Academic in the University of Newcastle, Newcastle, Australia. His research interest includes estimation, identification, and machine learning. 


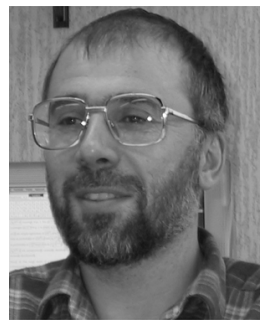

Rik Pintelon (M'90-SM'96-F'98) was born in Gent, Belgium, on December 4, 1959. He received the degree of electrical engineer (burgerlijk ingenieur) in 1982, the degree of doctor in applied sciences in 1988, and the qualification to teach at the university level (geaggregeerde voor het hoger onderwijs) in 1994, all from the Vrije Universiteit Brussel (VUB), Brussels, Belgium.

From October 1982 to September 2000, he was a Researcher of the Fund for Scientific Research - Flanders at the VUB. Since October 2000, he has been a

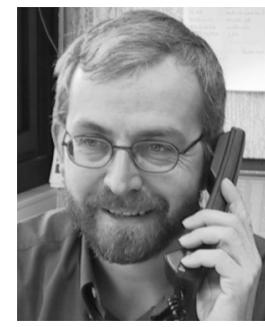

Johan Schoukens (M'90-SM'92-F'97) received the degree of engineer in 1980 and the degree of doctor in applied sciences in 1985, both from the Vrije Universiteit Brussel (VUB), Brussels, Belgium.

He is currently a Professor at the VUB. The prime factors of his research are in the field of system identification for linear and nonlinear systems.

He received from the IEEE Instrumentation and Measurement Society the Best Paper Award (2002) and the Society Distinguished Service Award (2003).

Professor at the VUB in the Electrical Measurement Department (ELEC). His main research interests are in the fields of parameter estimation/system identification and signal processing. 